\title{
Features of forward $\pi N$ scattering from a Reggeized model
}

\author{
Kook-Jin Kong* and Byung-Geel Yu ${ }^{\dagger}$ \\ Research Institute of Basic Science, Korea Aerospace University, Koyang 10540, Korea
}

(Received 17 August 2018; published 19 October 2018)

\begin{abstract}
Charge exchange process $\pi^{-} p \rightarrow \pi^{0} n$ and elastic scatterings $\pi^{ \pm} p \rightarrow \pi^{ \pm} p$ are investigated within the Regge framework where the relativistic Born amplitude is Reggeized for the $t$-channel meson exchange. The charge exchange cross section is featured by single $\rho$ exchange. Additional corrections by Regge cuts, $\rho$ - $f_{2}$ and $\rho$-Pomeron, agree with differential cross sections, and a new trajectory for the $\rho^{\prime}(1450)$ exchange is attempted to reproduce polarization data. For the description of elastic scattering data up to pion momentum $P_{\mathrm{Lab}} \approx 250 \mathrm{GeV} / \mathrm{c}$, Pomeron exchange of the Donnachie-Landshoff type is newly constructed and applied in this work. Elastic cross section data are well reproduced with the dominance of $f_{2}$ and Pomeron exchanges in intermediate and high energies. Analysis of nucleon resonances is presented to test the validity of the present Regge framework below $W \leqslant 2 \mathrm{GeV}$.
\end{abstract}

DOI: 10.1103/PhysRevC.98.045207

\section{INTRODUCTION}

A $\pi N$ system is one of the fundamental objects for understanding strong interaction with its origin from QCD. The $\pi N$ scattering near threshold offers a testing ground for the chiral dynamics of QCD in terms of soft pion interaction [1]. On the other hand, the rich structure of nucleon resonances $\Delta$ and $N^{*}$ in $\pi N$ scattering below the reaction energy $2 \mathrm{GeV}$ strongly supports the quark model prediction for the baryonic spectrum and its properties [2-4]. Over the resonance region up to hundreds of $\mathrm{GeV}$ the reaction provides information on various meson exchanges and the nonresonant diffractive scattering that could be a manifestation of quark and gluon degrees of freedom rather than hadronic degrees of freedom [5]. Therefore, though not listing a long history of theoretical development and experimental activities initiated by $\pi N$ scattering, the reaction should be regarded as an important source for understanding the dynamics of QCD in the isospin symmetry sector.

Recently, Mathieu et al. [6] of the Joint Physics Analysis Center (JPAC) studied $\pi N$ scattering to construct a new set of Regge amplitudes by matching the low-energy partial wave analysis with high-energy data via the finite energy sum rule. Nys et al. of JPAC also analyzed world data of $K N$ charge exchange reactions with beam energy above $5 \mathrm{GeV} / c$ [7]. Huang et al. [8] investigated $\pi N$ charge exchange scattering by using the Regge-cut model to provide high-energy constraints above

\footnotetext{
*kong@kau.ac.kr

†bgyu@kau.ac.kr
}

Published by the American Physical Society under the terms of the Creative Commons Attribution 4.0 International license. Further distribution of this work must maintain attribution to the author(s) and the published article's title, journal citation, and DOI. Funded by $S C O A P^{3}$.
$2 \mathrm{GeV}$ for the analysis of baryon resonances. The primary interest of these works is in the knowledge of $\pi N$ and $K N$ scatterings at high energy in order to provide a supplementary method for an extraction of properties of nucleon resonances in the low-energy region. However, the information obtained from these analyses is less straightforward for current model calculations based on the effective Lagrangian approach, such as the standard baryon pole model [9], because the residues in the $t$-channel helicity Regge poles fitted to empirical data in Refs. [6-8] cannot communicate with coupling strengths of hadron interactions in the Lagrangian formalism.

Therefore, it is desirable to investigate $\pi N$ scattering with hadron models that can utilize the effective Lagrangians for the description of the reaction beyond resonances up to the pion momentum $P_{\text {Lab }} \approx 250 \mathrm{GeV} / c$, the highest energy where a data point exists. Unfortunately, however, there is no theory, and no model calculations at present are available for such a purpose.

In this paper, we investigate $\pi N$ charge exchange and elastic scatterings for the analysis of the reaction mechanism by the peripheral process. To accomplish this, we construct the Born amplitude to be Reggeized for the meson exchange, with our interest being to establish the reaction amplitude up to such high momentum with the interaction Lagrangians and the coupling constants shared with other hadron reactions. Another issue to be addressed here is to provide the Pomeron exchange that could be well suited for the reaction amplitude thus constructed. The quark-Pomeron coupling picture is introduced to $\pi N$ elastic scattering, similar to the case of photoproductions of neutral vector mesons [5,10-13]. Therefore, complementary to previous findings in Refs. [6-8], the result of this work may serve to complete of our understanding the reaction mechanism of $\pi N$ scattering beyond resonances.

The paper is organized as follows. In Sec. II we begin with a statement of phenomenological features of charge exchange scattering $\pi^{-} p \rightarrow \pi^{0} n$ to construct the Regge amplitude for the $t$-channel $\rho$-meson exchange. To account for the dip in 
the differential cross section we introduce Regge cuts [14], and to reproduce polarization asymmetry we consider a new trajectory $\rho(1450)$. Section III follows steps similar to those in the preceeding section. Features of elastic scattering process $\pi^{ \pm} p \rightarrow \pi^{ \pm} p$ are introduced and the Regge pole amplitudes relevant to these reactions are fully constructed. The implementation of the Pomeron exchange from the quark picture $[5,10,11,15,16]$ is presented. We present numerical results in experimental data at high energies for total and differential cross sections as well as polarization asymmetry. Section IV is devoted to an incorporation of nucleon resonances with the Regge poles in the $t$ channel in the $\pi N$ scattering. We discuss the Breit-Wigner form of the nucleon resonance in the multipole expansion of the scattering amplitude, for application to the energy region below $W \leqslant 2 \mathrm{GeV}$. In Sec. V we discuss our findings and give a summary with conclusions.

\section{CHARGE EXCHANGE SCATTERING}

\section{A. General features}

By charge conservation and isospin symmetry the charge exchange reaction $\pi^{-} p \rightarrow \pi^{0} n$ allows only the single $\rho$ exchange in the $t$ channel. Thus, it is natural to expect that differential cross sections would show a dip structure at the nonsense wrong signature zero (NWSZ) of $\rho$ trajectory $-t \approx 0.5(\mathrm{GeV} / c)^{2}$ from $\alpha_{\rho}(t)=0$. Moreover, polarization asymmetries in this process should appear to be vanishing, because the polarization asymmetry $P(\theta)$ is defined as the interference between the spin nonflip and flip amplitudes, i.e.,

$$
P(\theta)=\frac{2 \operatorname{Im}\left[\mathcal{M}^{++} \mathcal{M}^{+-*}\right]}{\left|\mathcal{M}^{++}\right|^{2}+\left|\mathcal{M}^{+-}\right|^{2}},
$$

and the single $\rho$ exchange, which gives a dominant contribution to the spin flip amplitude, cannot produce nontrivial phase interference between them. Therefore, the reaction needs more theoretical consideration, such as Regge cuts and other meson exchanges in the $t$ channel, to reproduce the differential cross section data and polarization asymmetry at small angles.

\section{B. Regge description}

For the reaction $\pi(k)+N(p) \rightarrow \pi(q)+N\left(p^{\prime}\right)$ process, we denote the incoming and outgoing pion momenta by $k$ and $q$, and the initial and final nucleon momenta by $p$ and $p^{\prime}$, respectively. Then, conservation of four-momentum requires $k+p=q+p^{\prime}$, and $s=(k+p)^{2}, t=(q-k)^{2}$, and $u=\left(p^{\prime}-k\right)^{2}$ are the invariant Mandelstam variables. The total energy $W$ is related with the pion momentum in the laboratory system, $P_{\mathrm{Lab}}$, by the equation $W=$ $\sqrt{M^{2}+m_{\pi}^{2}+2 M \sqrt{P_{\mathrm{Lab}}^{2}+m_{\pi}^{2}}}$ with $M$ and $m_{\pi}$ the nucleon and pion masses. The relevant expressions for the momenta in the laboratory frame and c.m. frame are defined as

$$
\begin{aligned}
P_{\mathrm{Lab}} & =\sqrt{\left(\frac{s-M^{2}-m_{\pi}^{2}}{2 M}\right)^{2}-m_{\pi}^{2}}, \\
P_{c . m .} & =\frac{1}{\sqrt{2 s}} \sqrt{\left(s-\left(M+m_{\pi}\right)^{2}\right)\left(s-\left(M-m_{\pi}\right)^{2}\right)},
\end{aligned}
$$

respectively.

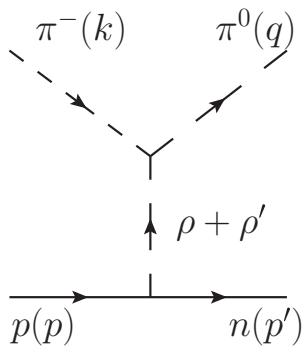

(a)

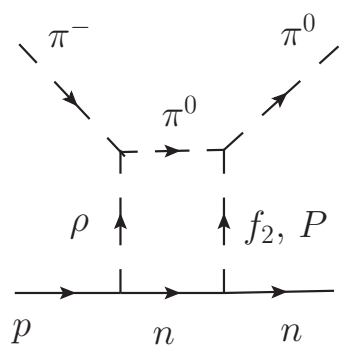

(b)
FIG. 1. Reaction process for $\pi^{-} p \rightarrow \pi^{0} n$ in the $t$ channel. (a) $\rho(775)$ and $\rho^{\prime}(1450)$ Regge pole exchanges. (b) Elastic cuts $\rho-f_{2}$ and $\rho$-Pomeron.

Let us begin with the scattering amplitude simply given by the single $\rho$ exchange,

$$
\mathcal{M}\left(\pi^{-} p \rightarrow \pi^{0} n\right)=-\sqrt{2} \mathcal{M}_{\rho},
$$

and the Born amplitude in Fig. 1 relevant to the $t$-channel $\rho$-meson exchange, written as

$$
\mathcal{M}_{\rho}=\Gamma_{\rho \pi \pi}^{\mu}(q, k) \frac{\Pi_{\mu \nu}^{\rho}(Q)}{t-m_{\rho}^{2}} \Gamma_{\rho N N}^{\nu}\left(p^{\prime}, p\right),
$$

with $Q=q-k$ the $t$-channel momentum transfer. The coupling vertices with spin polarization $\Pi_{\rho}^{\mu v}$ are expressed as

$$
\begin{aligned}
\Gamma_{\rho \pi \pi}^{\mu}(q, k) & =g_{\rho \pi \pi}(q+k)^{\mu}, \\
\Gamma_{\rho N N}^{v}\left(p^{\prime}, p\right) & =\bar{u}\left(p^{\prime}\right)\left[g_{\rho N N}^{v} \gamma^{\nu}+\frac{g_{\rho N N}^{t}}{4 M}\left[\gamma^{\nu}, Q\right]\right] u(p), \\
\Pi_{\rho}^{\mu \nu}(Q) & =-g^{\mu \nu}+Q^{\mu} Q^{\nu} / m_{\rho}^{2},
\end{aligned}
$$

from the Lagrangians for $\rho \pi \pi$ and $\rho N N$ interactions,

$$
\begin{aligned}
\mathcal{L}_{\rho \pi \pi} & =g_{\rho \pi \pi} \vec{\rho}_{\mu} \cdot\left(\vec{\pi} \times \partial^{\mu} \vec{\pi}\right), \\
\mathcal{L}_{\rho N N} & =\bar{N}\left[g_{\rho N N}^{v} \gamma_{\nu}-i \frac{g_{\rho N N}^{t}}{2 M} \sigma_{\mu \nu} \partial^{v}\right]\left(\tau_{a}\right) \rho^{v} N .
\end{aligned}
$$

In order to describe the reaction at high energies up to tens of $\mathrm{GeV}$, we make the above Born amplitude Reggeized by replacing the Feynman propagator with the Regge one,

$$
\frac{1}{t-m_{\varphi}^{2}} \rightarrow \mathcal{R}^{\varphi}(s, t),
$$

where the Regge propagator is written as

$$
\mathcal{R}^{\varphi}(s, t)=\frac{\pi \alpha_{J}^{\prime} \times \text { phase }}{\Gamma\left[\alpha_{J}(t)+1-J\right] \sin \left[\pi \alpha_{J}(t)\right]}\left(\frac{s}{s_{0}}\right)^{\alpha_{J}(t)-J}
$$

for the $\varphi$ meson of spin $J$ and $s_{0}=1 \mathrm{GeV}^{2} . \varphi$ stands for $\rho$ here, and $\omega, \sigma$, and $f_{2}$ collectively for later use. The trajectory of spin- $J$ meson is denoted by $\alpha_{J}(t)$. The phase factor is, in general, taken to be $\frac{1}{2}\left[(-1)^{J}+e^{-i \pi \alpha_{J}(t)}\right]$ for the exchange nondegenerate meson exchange.

From Eq. (6) the decay width is evaluated as

$$
\Gamma(\rho \rightarrow \pi \pi)=\frac{g_{\rho \pi \pi}^{2} k^{3}}{6 \pi m_{\rho}^{2}},
$$




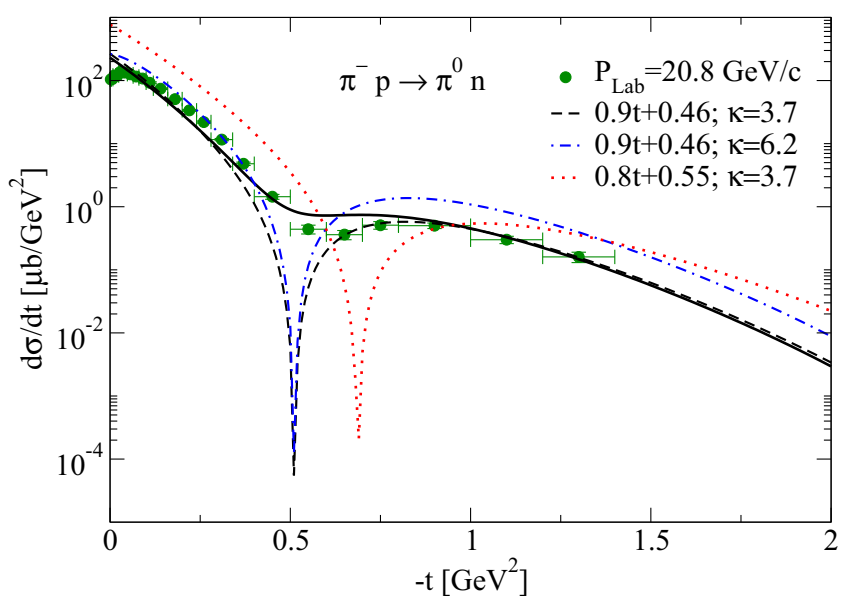

FIG. 2. Differential cross section $d \sigma / d t$ for $\pi^{-} p \rightarrow \pi^{0} n$ scattering at $P_{\mathrm{Lab}}=20.8 \mathrm{GeV} / c$. The cross section from the single $\rho$ exchange with $\kappa_{\rho}=3.7$ and $0.9 t+0.46$ is given by the dashed curve, while the case with the Regge cuts, $\rho+\left(\rho-f_{2}+\rho-\mathbb{P}\right)$, is denoted by the solid curve. For comparison we present cross sections from the single $\rho$ exchange with $\kappa_{\rho}=3.7$ and $0.8 t+0.55$ (red dotted) and with $\kappa_{\rho}=6.2$ and $0.9 t+0.46$ (blue dash-dotted). Data are taken from Ref. [19]. The additional contribution of $\rho(1450)$ does not alter the above results.

which leads to $g_{\rho \pi \pi}=5.95$ from $\Gamma\left(\rho \rightarrow \pi^{+} \pi^{-}\right)=147.8$ $\mathrm{MeV}$ reported by the Particle Data Group (PDG). For the $\rho N N$ coupling constants, we use $g_{\rho N N}^{v}=2.6$ throughout this work for consistency with our previous works on photoproductions of hadrons. We choose $g_{\rho N N}^{t}=9.62$ to be consistent with the vector meson dominance (VMD) for the anomalous magnetic moment $\kappa_{\rho}=3.7$. However, the universality of the $\rho$ meson coupling constant is not exact between $g_{\rho \pi \pi}$ and $2 g_{\rho N N}^{v}$, the latter of which is estimated from the $\rho$ meson decay width $\Gamma\left(\rho^{0} \rightarrow e^{+} e^{-}\right)$.

Given the coupling constants above we take $\alpha_{\rho}(t)=$ $0.9 t+0.46$ from the Regge analyses of charged $\rho$ photoproductions $[17,18]$ together with the exchange nondegenerate phase for the $\rho$ Regge pole to reproduce the differential cross section at $P_{\mathrm{Lab}}=20.8 \mathrm{GeV} / c$. The result is presented in Fig. 2 by the dashed curve.

In modeling hadron reactions involved in the $\rho$-meson coupling to the nucleon there is another option for $\kappa_{\rho}=6.2$ from the analysis of $N N$ potential. Also the trajectory $\alpha_{\rho}=0.8 t+$ 0.55 is frequently employed with a stronger coupling constant $2 g_{\rho N N}^{v} \approx g_{\rho \pi \pi}$ in Regge model calculations [20]. Without any model parameters such as cutoff masses of form factors in this work, the model dependence lies in the Regge trajectories and meson-baryon coupling constants chosen. Thus, by taking the advantage of the single $\rho$ dominance of the reaction, it is interesting to ask what are the proper choices for the $\rho$-meson coupling constants and trajectory. For comparison we show the (red) dotted curve resulting from the case of $\rho$ trajectory $0.8 t+0.55$ with $\kappa_{\rho}=3.7$ and the (blue) dash-dotted one from $0.9 t+0.46$ with $\kappa_{\rho}=6.2$. It is clear that both cross sections are overestimating experimental data. Moreover, the dip position at the NWSZ, $-t \approx 0.69(\mathrm{GeV} / c)^{2}$ in the case of $0.8 t+0.55=0$, deviates from data. These findings in the $\pi^{-} p \rightarrow \pi^{0} n$ reaction support the validity of $\alpha_{\rho}=0.9 t+$ 0.46 with $\kappa_{\rho}=3.7$ as much as in our previous study on the charged $\rho$ photoproductions $[17,18]$.

\section{1. $\rho$ cuts}

As discussed above, we now find a way to fill up the deep dip in the differential cross section in Fig. 2. Similar to neutral pion photoproduction $\gamma p \rightarrow \pi^{0} p[14,21]$, the $\rho$ cuts are introduced for this purpose, and the reaction amplitude in Eq. (4) is now extended to be [13]

$$
\begin{aligned}
\mathcal{M}_{\rho}= & \Gamma_{\rho \pi \pi}^{\mu}(q, k) \Pi_{\mu \nu}^{\rho}(Q) \Gamma_{\rho N N}^{\nu}\left(p^{\prime}, p\right) \\
& \times\left[\mathcal{R}^{\rho}(s, t)+\sum_{\varphi} C_{\varphi} e^{d_{\varphi} t} e^{-i \pi \alpha_{c}^{\varphi}(t) / 2}\left(\frac{s}{s_{0}}\right)^{\alpha_{c}^{\varphi}(t)-1}\right],
\end{aligned}
$$

where $\varphi=f_{2}$ and $\mathbb{P}$ are the subsequent Regge pole exchanges following the $\rho$ exchange in the elastic cut, as shown in Fig. 1(b). The cut parameters $C_{\varphi}$ and $d_{\varphi}$ represent the strength and range of the cut to be fitted to experimental data. The cut trajectory for $\rho-\varphi$ is given by a composite of two trajectories, i.e.,

$$
\alpha_{c}^{\varphi}=\frac{\alpha_{\rho}^{\prime} \alpha_{\varphi}^{\prime}}{\alpha_{\rho}^{\prime}+\alpha_{\varphi}^{\prime}} t+\left[\alpha_{\rho}(0)+\alpha_{\varphi}(0)-1\right]
$$

with its slope and intercept consisting of each slope and intercept. Here we use the tensor meson $f_{2}$ trajectory $\alpha_{f_{2}}(t)=$ $0.9 t+0.53$, sharing with the $\omega$ trajectory. The Pomeron trajectory is determined as $\alpha_{\mathbb{P}}(t)=0.12 t+1.06$ by the fit of elastic scattering data at high momenta $P_{\mathrm{Lab}}=100$ and $200 \mathrm{GeV} / c$. We shall discuss this point in Sec. III later.

In Fig. 2 the differential cross section shows the cut effect to fill up the dip by the $\rho$ exchange with parameters $C_{f_{2}}=$ $1.0 \mathrm{GeV}^{-2}, d_{f_{2}}=2 \mathrm{GeV}^{-2}$ for $\rho-f_{2}$, and $C_{\mathbb{P}}=0.1 \mathrm{GeV}^{-2}$, $d_{\mathbb{P}}=5 \mathrm{GeV}^{-2}$ for $\rho-\mathbb{P}$. Such an agreement with experimental data as can be seen in Fig. 2 appears repeatedly in differential cross sections at other momenta at the same level of quality.

Polarization asymmetry $P$ in the $\pi N$ scattering is the observable that could validate the accuracy of model predictions. As defined in Eq. (1), it arises via the interference between exchanges of different mesons. It is obvious that the $\rho$ cuts could give rise to no interference, though they are added because they share the same interaction vertices with the single $\rho$ as in Eq. (14). Thus, we consider another isovector exchange in the $t$ channel to find that $\rho(1450)$, with the same quantum number $1^{+}\left(1^{--}\right)$as $\rho(775)$ but with higher mass, could be a candidate to produce nonvanishing polarization.

\section{Daughter trajectory $\rho^{\prime}(1450)$}

To induce the phase interference between two different Regge poles, we consider the daughter trajectory of a $\rho$ meson of higher mass $m_{\rho^{\prime}}=1450 \mathrm{MeV}$ [22], with the $\pi \pi$ decay mode evident but not measured yet. [For distinction we denote $\rho(1450)$ by $\rho^{\prime}$.] Therefore, no information is available for the $\rho^{\prime}$ coupling to $\pi$ or to the nucleon. We treat these coupling constants as parameters to fit to polarization data. The trajectory relevant to $\rho^{\prime}(1450)$ is calculated from the relativistic 

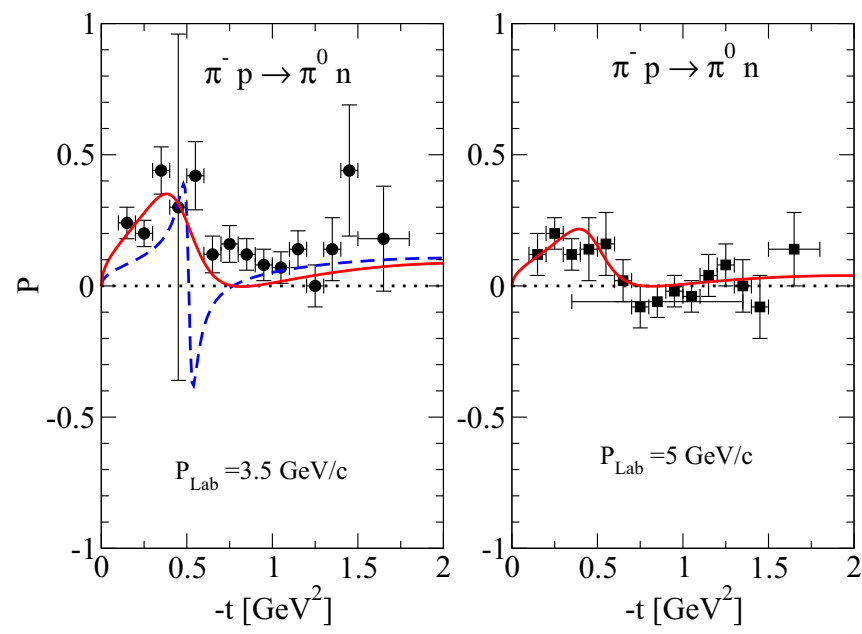

FIG. 3. Polarizations for $\pi^{-} p \rightarrow \pi^{0} n$ scattering versus $-t$ at $P_{\mathrm{Lab}}=3.5$ and $5 \mathrm{GeV} / c$. Dotted curves are the polarizations from $\rho+$ cuts without $\rho^{\prime}(1450)$. The dashed curve is from $\rho+\rho^{\prime}$ without cuts. Data are taken from Ref. [24].

quark model in Ref. [23] to be $\alpha_{\rho^{\prime}}(t)=t-1.23$, which is different from that of $\rho(775)$. Thus, the amplitude in Eq. (4) is extended to include the amplitude $\mathcal{M}_{\rho^{\prime}}$ of the same form as in Eq. (5) in addition to Eq. (14). In the calculation we use both the $\rho$ and $\rho^{\prime}$ trajectory exchanges nondegenerate. Since the intercept of the $\rho^{\prime}$ trajectory is very low, the $\rho^{\prime}$ exchange gives no contribution, significantly altering the differential cross section at high momentum as in Fig. 2. Nevertheless, the interference of phases between $\rho^{\prime}$ and $\rho$ cuts could reproduce the polarization data to a good degree.

Figure 3 shows the polarization measured at $P_{\mathrm{Lab}}=3.5$ and $5 \mathrm{GeV} / c$ in the range $0.2 \leqq-t \leqq 1.8(\mathrm{GeV} / c)^{2}$, where the solid curve is prediction by the full amplitude

$$
-\sqrt{2}\left[\rho+\rho-\text { cuts }+\rho^{\prime}(1450)\right]
$$

At the choice of $\rho^{\prime}(1450)$ coupling constants, $G_{\rho^{\prime}}^{v}=40$ and $G_{\rho^{\prime}}^{t}=-75$ with $G_{\rho^{\prime}}^{v}=g_{\rho^{\prime} \pi \pi} g_{\rho^{\prime} N N}^{v}, G_{\rho^{\prime}}^{t}=g_{\rho^{\prime} \pi \pi} g_{\rho^{\prime} N N}^{t}$, we obtain a quite good fit of the polarization data. Of course, adjusting these values leads to some change of the polarization in magnitude around $-t \approx 0.5(\mathrm{GeV} / c)^{2}$, but not in shape, unless the signs of coupling constants are changed. The dotted curves are from the single $\rho(775)$ showing null polarizations as discussed. The dashed curve results from the $\rho(775)$ with $\rho^{\prime}(1450)$, but without $\rho$ cuts. Though dependent on the coupling constants $G_{\rho^{\prime}}^{v}$ and $G_{\rho^{\prime}}^{t}$, we recognize the implication of the $\rho$ cuts and daughter $\rho^{\prime}$ which result in an agreement with vanishing of the polarization at $-t \approx 0.7(\mathrm{GeV} / c)^{2}$ and a slow increase as the $-t$ becomes larger.

We present the total cross section in Fig. 4 for comparison with experimental data from threshold up to $W=10 \mathrm{GeV}$. The single $\rho$ exchange is given by the dotted curve and the case of $\rho(775)+\rho^{\prime}(1450)$ by the dashed curve. The cross section from the full amplitude $\rho+\rho^{\prime}+\rho$ cuts is depicted by the solid curve.

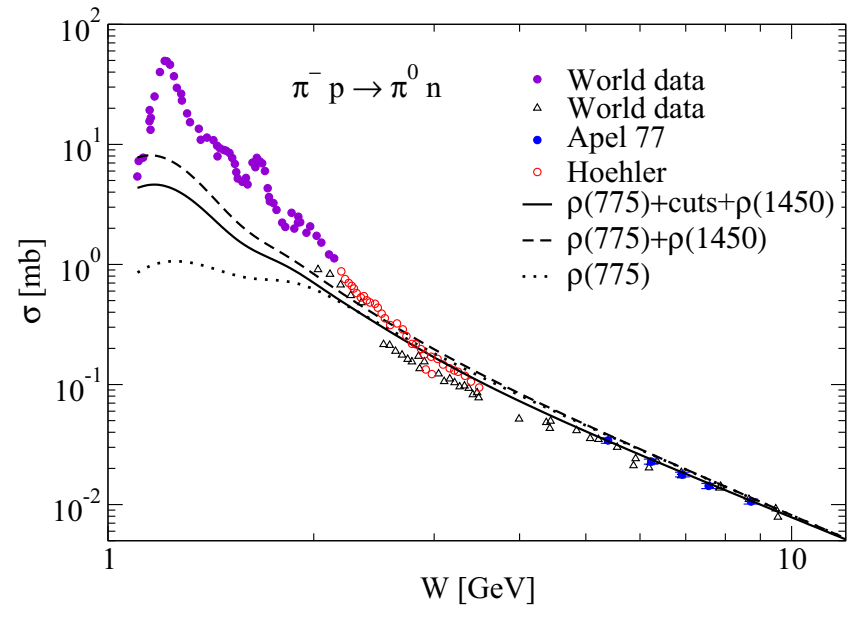

FIG. 4. Total cross section for $\pi^{-} p \rightarrow \pi^{0} n$ as a function of invariant energy $W$. Data show the resonance peaks below $W \approx$ $2 \mathrm{GeV}$. Theory and experiment coincide over the resonance region. Data are taken from Refs. [8,25].

\section{ELASTIC SCATTERING PROCESS}

\section{A. General features}

Elastic scatterings $\pi^{ \pm} p \rightarrow \pi^{ \pm} p$ proceed via the $s$-channel $\Delta$ and $N^{*}$ excitations to show the prominent resonance structures around $W \approx 1.2$ and $1.6 \mathrm{GeV}$, respectively. Over the resonances the peripheral scattering of the $t$-channel meson exchange dominates the reactions to gradually exhibit a slow increase as the reaction energy increases, i.e., the diffraction scattering which is a manifestation of the Pomeron exchange in hadron elastic reactions. Differential cross sections have smooth $t$ dependence and no dips there. The sign of nucleon spin polarization at very small angle is consistent with the sign of pion charge in the $\pi^{ \pm} p$ reactions.

\section{B. Regge description}

Previous studies of the $\pi N$ reaction were devoted to the analysis of total cross section, which includes all the inelastic subprocesses in addition to the elastic one $[6,26,27]$. In the conventional approach where the residues are fitted to scattering data in the $t$-channel helicity Regge poles, the role of $\rho$ exchange was expected to account for the difference of total cross sections between the $\pi^{+} p$ and $\pi^{-} p$ reactions in addition to the Pomeron and the second vacuum exchange at high energy [26]. Recent models of JPAC [6,7] improved the Regge amplitude to include tensor meson $f_{2}$ exchange instead of the second vacuum exchange.

In contrast to these works, however, the exclusive elastic reactions for $\pi^{ \pm} p \rightarrow \pi^{ \pm} p$ are the main topics of the present section to be investigated in the Reggeized Born term model, as in the previous section. The Lagrangian formulation of hadron interactions in the model requires those mesons that are decaying to $\pi \pi$ in the $t$-channel exchange. In this respect exchanges of scalar meson and vector meson $\omega$ are further included in the present work. The Pomeron exchange is viewed from the quark-Pomeron coupling picture and we construct a 
new amplitude, which is of Donnachie and Landshoff (DL) type rather than the original version by Pichowsky [5].

As advertised, the reaction amplitude that contains all the mesons decaying to two pions is written as

$$
\mathcal{M}\left(\pi^{ \pm} p\right)=\mathcal{M}_{\sigma} \mp \mathcal{M}_{\omega} \mp \mathcal{M}_{\rho}+\mathcal{M}_{f_{2}}+\mathcal{M}_{\mathbb{P}},
$$

where the vector mesons of $C$ parity odd change sign in accordance with pion charge. Thus, the two channels with opposite charges $\pi^{+} p$ and $\pi^{-} p$ are distinguished by the roles of the $\omega$ - and $\rho$-meson exchange. For the sake of consistency we share the meson-baryon coupling constants and the Regge trajectories in the $\pi N$ scattering with those used for meson photoproduction.

\section{Vector mesons $\rho$ and $\omega$ exchanges}

Given the $\rho$ Regge pole in the previous section, we include the Reggeized $\omega$ exchange in the same form as in Eq. (5) with the propagator in Eq. (12). The $\omega \pi \pi$ coupling is estimated to be $g_{\omega \pi \pi}= \pm 0.18$ from the decay width with $\Gamma\left(\omega \rightarrow \pi^{+} \pi^{-}\right)=0.13 \mathrm{MeV}$ taken from PDG. We use $g_{\omega N N}^{v}=15.6$ and $\kappa_{\omega}=0$ for the $\omega N N$ couplings. The trajectory $\alpha_{\omega}(t)=0.9 t+0.44$ constitutes a degenerate pair with the $f_{2}$ trajectory so that both the $\omega$ and $f_{2}$ Regge poles share the exchange degenerate phase in common. For a phenomenologically better description we adopt the constant phase for both reactions $\pi^{ \pm} p \rightarrow \pi^{ \pm} p$.

\section{Scalar meson $\sigma$ exchange}

The mass and full width of scalar meson $\sigma$ are reported to be $m_{\sigma}=400-550 \mathrm{MeV}$ and $\Gamma=400-700 \mathrm{MeV}$ in the PDG. The scalar meson $\sigma$ is the lightest meson to exchange, so it could contribute to the threshold behavior of reaction cross sections for $\pi^{ \pm} p$ elastic scattering.

The interaction Lagrangians relevant to the coupling of $\sigma$ meson to hadrons are given by

$$
\begin{aligned}
& \mathcal{L}_{S}=-\frac{1}{2} g_{\sigma \pi \pi} m_{\pi} \sigma \vec{\pi} \cdot \vec{\pi}-g_{\sigma N N} \sigma \bar{N} N, \\
& \mathcal{L}_{V}=\frac{f_{\sigma \pi \pi}}{2 m_{\pi}} \sigma \partial_{\mu} \vec{\pi} \cdot \partial^{\mu} \vec{\pi}+g_{\sigma N N} \sigma \bar{N} N,
\end{aligned}
$$

where the scalar and vector couplings are considered for $\sigma \pi \pi$ coupling in a manner consistent with the scalar meson $\sigma$ as the two-pion $s$-wave correlation. However, the uncertainty in the broad decay width makes our estimate of the $\sigma \pi \pi$ coupling constant very model dependent. A naive estimate of $g_{\sigma \pi \pi}$ by taking $\Gamma(\sigma \rightarrow \pi \pi)=400 \mathrm{MeV}$, for instance, from the decay width

$$
\Gamma\left(\sigma \rightarrow \pi^{+} \pi^{-}\right)=\frac{2}{3} \Gamma(\sigma \rightarrow \pi \pi)=\frac{g_{\sigma \pi \pi}^{2} m_{\pi}^{2} k}{12 \pi m_{\sigma}^{2}},
$$

yields the value $g_{\sigma \pi \pi}= \pm 20.37$, which is larger than the value $7.91-16.54$ extracted from the $J / \psi$ decay $[28,29]$. Here, the factor $2 / 3$ is taken into account for charged channels in the isospin space.

We now write the Born amplitude for $\sigma$ exchange as

$$
\mathcal{M}_{\sigma}=\Gamma_{\sigma \pi \pi}^{S / V}(q, k) \frac{1}{t-m_{\sigma}^{2}} \Gamma_{\sigma N N}\left(p^{\prime}, p\right),
$$

where the scalar and vector coupling vertices are given by

$$
\begin{aligned}
& \Gamma_{\sigma \pi \pi}^{S}(q, k)=g_{\sigma \pi \pi} m_{\pi}, \\
& \Gamma_{\sigma \pi \pi}^{V}(q, k)=\frac{f_{\sigma \pi \pi}}{m_{\pi}} q \cdot k,
\end{aligned}
$$

and

$$
\Gamma_{\sigma N N}\left(p^{\prime}, p\right)=g_{\sigma N N} \bar{u}\left(p^{\prime}\right) u(p),
$$

for the $\sigma$-meson nucleon coupling vertex.

The $\sigma$-meson exchange as the two-pion correlation in the $s$ state with a broad width was studied in the $\pi \pi \rightarrow N \bar{N}$ reaction [30]. In the $\pi N$ scattering, as a result, the $t$-channel $\sigma$ pole derived from the dispersion relation is expressed as

$$
\mathcal{M}_{\sigma}=\bar{u}\left(p^{\prime}\right) g_{\sigma}(t) \frac{t-2 m_{\pi}^{2}}{m_{\sigma}^{2}-t} u(p),
$$

which corresponds to

$$
\begin{aligned}
& g_{\sigma}(t)=\frac{g_{\sigma N N} g_{\sigma \pi \pi} m_{\pi}}{2 q \cdot k}, \\
& g_{\sigma}(t)=\frac{g_{\sigma N N} f_{\sigma \pi \pi}}{2 m_{\pi}},
\end{aligned}
$$

for the scalar and vector couplings in Eq. (21), respectively. These results imply that the $\sigma$ pole with the large decay width term, $1 /\left(t-m_{\sigma}^{2}+i \Gamma_{\sigma} m_{\sigma}\right)$, in the pole model can be equivalently expressed as in Eq. (25) from the dispersion relation. In Eqs. (26) and (27), while the latter term remains constant, the former has the energy dependence $1 /(q \cdot k)$, which is singular at threshold. Therefore, we favor to adopt the vector coupling scheme for $\sigma$ exchange in Eq. (21) with the coupling constant $f_{\sigma \pi \pi}$ properly chosen to describe cross section data near threshold. In the calculation we use $g_{\sigma N N}=$ 14.6 for consistency with the photoproduction of neutral vector mesons $[12,13]$.

\section{Tensor meson $f_{2}$ exchange}

For the $f_{2}$ tensor meson exchange, we use the following interaction Lagrangian:

$$
\mathcal{L}_{f_{2} \pi \pi}=\frac{2 g_{f_{2} \pi \pi}}{m_{f_{2}}} \partial_{\mu} \vec{\pi} \cdot \partial_{\nu} \vec{\pi} f^{\mu \nu}
$$

for the $f_{2} \pi \pi$ coupling, with $f^{\mu v}$ the spin- 2 tensor meson field. This gives the coupling vertex

$$
e_{\mu \nu} \Gamma_{f_{2} \pi \pi}^{\mu \nu}=\frac{g_{f_{2} \pi \pi}}{m_{f_{2}}}(k+q)^{\mu}(k+q)^{v} e_{\mu \nu}
$$

with $e^{\mu \nu}$ the spin-2 polarization tensor. The decay width for $f_{2} \rightarrow \pi \pi$ is given by

$$
\Gamma\left(f_{2} \rightarrow \pi^{+} \pi^{-}\right)=\frac{2}{3} \Gamma\left(f_{2} \rightarrow \pi \pi\right)=\frac{4 g_{f_{2} \pi \pi}^{2}}{15 \pi} \frac{p^{5}}{m_{f_{2}}^{4}},
$$

where $p=\sqrt{m_{f_{2}}^{2} / 4-m_{\pi}^{2}}$ is the momentum of the $\pi$ meson. From the full width in the range $121 \lesssim \Gamma\left(f_{2}\right) \lesssim 240 \mathrm{MeV}$ in PDG with the branching fraction $84.2 \%$ for the $f_{2} \rightarrow \pi \pi$, the coupling constant is estimated to be in the range $4.76 \lesssim$ $g_{f_{2} \pi \pi} \lesssim 6.71$ in units of $m_{f_{2}}^{-1}$. 
TABLE I. The physical constants and Regge trajectories with the corresponding phase factors for $\pi^{ \pm} p \rightarrow \pi^{ \pm} p$. The symbol $\varphi$ stands for $\sigma, \omega, f_{2}$, and $\rho$. For the $\sigma$ couplings $g_{\varphi \pi \pi}$ should be understood as the vector coupling constant $f_{\sigma \pi \pi}$.

\begin{tabular}{lcccc}
\hline \hline Meson & Trajectory $\left(\alpha_{\varphi}\right)$ & Phase factor & $g_{\varphi л \pi}$ & $g_{\varphi N N}^{1}\left(g_{\varphi N N}^{2}\right)$ \\
\hline$\rho$ & $0.9 t+0.46$ & $\left(-1+e^{-i \pi \alpha_{\rho}}\right) / 2$ & 5.95 & $2.6(9.62)$ \\
$\sigma$ & $0.7\left(t-m_{\sigma}^{2}\right)$ & $\left(1+e^{-i \pi \alpha_{\sigma}}\right) / 2$ & 0.5 & 14.6 \\
$\omega$ & $0.9 t+0.44$ & 1 & -0.18 & $15.6(0)$ \\
$f_{2}$ & $0.9 t+0.53$ & 1 & 4.5 & $6.45(0)$ \\
\hline \hline
\end{tabular}

The reaction amplitude for the $f_{2}$ exchange is written as

$$
\mathcal{M}_{f_{2}}=\Gamma_{f_{2} \pi \pi}^{\mu \nu}(q, k) \frac{\Pi_{\mu \nu ; \alpha \beta}^{f_{2}}(Q)}{t-m_{f_{2}}^{2}} \Gamma_{f_{2} N N}^{\alpha \beta}\left(p^{\prime}, p\right),
$$

where the tensor meson-nucleon coupling vertex and the polarization tensor for spin-2 propagation are given by

$$
\begin{aligned}
& \Gamma_{f_{2} N N}^{\alpha \beta}\left(p^{\prime}, p\right) \\
& \quad=\bar{u}\left(p^{\prime}\right)\left[\frac{2 g_{f_{2} N N}^{(1)}}{M}\left(P^{\alpha} \gamma^{\beta}+P^{\beta} \gamma^{\alpha}\right)+\frac{4 g_{f_{2} N N}^{(2)}}{M^{2}} P^{\alpha} P^{\beta}\right] u(p),
\end{aligned}
$$

and the spin projection operator for a spin-2 particle

$$
\Pi_{f_{2}}^{\mu \nu ; \alpha \beta}(Q)=\frac{1}{2}\left(\bar{g}^{\mu \alpha} \bar{g}^{\nu \beta}+\bar{g}^{\mu \beta} \bar{g}^{\nu \alpha}\right)-\frac{1}{3} \bar{g}^{\mu \nu} \bar{g}^{\alpha \beta}
$$

with

$$
\bar{g}^{\mu \nu}=-g^{\mu \nu}+Q^{\mu} Q^{v} / m_{f_{2}}^{2} .
$$

The tensor-meson nucleon coupling constants extracted from the tensor meson dominance were $g_{f_{2} N N}^{(1)}=2.12$ and $g_{f_{2} N N}^{(2)} \approx 0$. But the phenomenological information extracted from the dispersion relation as well as the partial wave analysis for $\pi N$ scattering suggested rather the scattered values for the $f_{2} N N$ coupling constants as discussed in Ref. [20], which showed $2.12 \lesssim g_{f_{2} N N}^{(1)} \lesssim 7.93$ and $g_{f_{2} N N}^{(2)} \approx 0$. In those meson photoproductions involving the tensor meson exchange we used $g_{f_{2} N N}^{(1)}=6.45$ and $g_{f_{2} N N}^{(2)}=0$ [31] to agree with empirical data. For the elastic scattering of the $\pi p \rightarrow \pi p$ reaction we resume using these values for the sake of consistency, and make a list of the coupling constants and trajectories with the corresponding phase factors in Table I.

\section{Pomeron exchange}

The quark-Pomeron coupling model, as depicted in Fig. 5, is based on the factorization of the exclusive $\pi N$ scattering amplitude in terms of the product of the $\pi \rightarrow q+\bar{q}$ fluctuation, the scattering of the $q \bar{q}$ system by the proton, and finally the $q \bar{q}$ hadronization into a pion. From the observation of total cross sections for $p p, \pi p$, and $K p$ reactions at high energies, Donnachie and Landshoff stated that the Pomeron couples to the separate valence quark inside a hadron rather than to the hadron as a whole, and the strength of the Pomeron coupling to a hadron is determined by the radius of the hadron. Therefore, assuming a quark-Pomeron coupling strength of

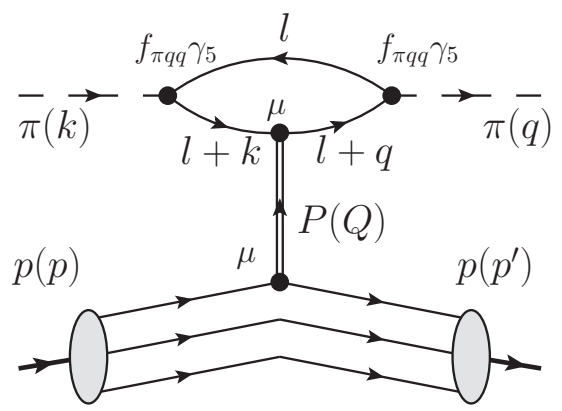

FIG. 5. Quark diagram for the Pomeron exchange in $\pi^{ \pm} p$ elastic scatterings. Pseudoscalar coupling $\pi \bar{q} i \gamma_{5} q$ with the coupling constant $f_{\pi q q}$ is assumed at the $\pi q \bar{q}$ coupling vertex. Momenta for quark loops are denoted by $l, l+k$, and $l+q$. The quark loop of momentum $l+k$ is off mass shell. Vector coupling $\gamma^{\mu}$ is used for the couplings of the Pomeron-quark and Pomeron-proton currents.

$F_{h}(t) \beta_{q} \gamma^{\mu}$ with the hadron form factor $F_{h}(t)$ for its size, the Pomeron contribution to the $\pi N$ cross section can be simply written as [10]

$$
\frac{d \sigma}{d t}=\frac{1}{4 \pi}\left|\left[2 \beta_{q} F_{\pi}(t)\right]\left(-i \alpha_{\mathbb{P}}^{\prime} s\right)^{\alpha_{\mathbb{P}}(t)-1}\left[3 \beta_{q^{\prime}} F_{1}(t)\right]\right|^{2}
$$

with the nucleon isoscalar form factor and pion form factor given by

$$
\begin{aligned}
& F_{1}(t)=\frac{4 M^{2}-2.8 t}{\left(4 M^{2}-t\right)\left(1-t / 0.71 \mathrm{GeV}^{2}\right)^{2}}, \\
& F_{\pi}(t)=\left(1-t / \Lambda^{2}\right)^{-n},
\end{aligned}
$$

and the Regge-type propagator

$$
\mathcal{R}^{\mathbb{P}}(s, t)=\left(\alpha_{\mathbb{P}}^{\prime} s\right)^{\alpha_{\mathbb{P}}(t)-1} e^{-i \frac{\pi}{2}\left[\alpha_{\mathbb{P}}(t)-1\right]} .
$$

Here $\alpha_{\mathbb{P}}(t)$ is the Pomeron trajectory of the form

$$
\alpha_{\mathbb{P}}(t)=\alpha_{\mathbb{P}}^{\prime} t+\alpha_{\mathbb{P}}^{0}
$$

A more rigorous treatment of the Pomeron exchange in the $\pi N$ elastic scattering can be found in Ref. [5] where the quark-meson coupling vertices in the incoming and outgoing states should be the Bethe-Salpeter amplitudes with the quark propagation arising from the Dyson-Schwinger equation for the bound state of the QCD. However, in the large-momentum limit, the current quark propagation could be replaced by the free quark (constituent quark) propagation with the constituent quark masses $m_{u(d)} \approx 330$ and $m_{s} \approx 490$ $\mathrm{MeV}$. Hence, the on-shell approximation for the quark loops of $l$ and $l+q$ for the outgoing pion, with the quark loop of $l+k$ considered to be off shell with the hadron form factor at the Pomeron- $\pi \pi$ vertex, is a good approximation to perform the loop integral [11]. In this work we follow the on-shell approximation as the Donnachie-Landshoff ansatz [32] for vector meson photoproduction [33], and use the pseudoscalar coupling

$$
f_{\pi q q} \bar{q} i \gamma_{5} q \pi
$$

for the $\pi q q$ vertex with the coupling strength $f_{\pi q q}$ in Fig. 5 .

The on-shell approximation leads to the loop integral simplification, and the trace calculation in the loop results in the 
following expression:

$$
\begin{aligned}
\operatorname{Tr} & {\left[\left(l+m_{q}\right) \gamma_{5}\left((l+\not k)+m_{q}\right) \gamma_{\mu}\left((l+q)+m_{q}\right) \gamma_{5}\right] } \\
& =-4 l \cdot q k_{\mu}-4 l \cdot k q_{\mu}+4 k \cdot q l_{\mu}, \\
& =m_{\pi}^{2}\left(k_{\mu}+q_{\mu}\right) .
\end{aligned}
$$

In the quark loop in Fig. 5 the two quarks in the outgoing pion state share the equal pion momentum $l=-q / 2$, with the assumption that they are nearly on shell. Then, the other quark loop of momentum $l+k$ in the figure is off shell and the propagator turns out to be

$$
\frac{1}{(l+k)^{2}-m_{q}^{2}}=\frac{-2}{2 m_{q}^{2}-m_{\pi}^{2} / 2-t}
$$

with $l=-q / 2$.

For the $\pi N$ elastic scattering, therefore, the Pomeron exchange is written as

$$
\begin{aligned}
\mathcal{M}_{\mathbb{P}}= & i 2 F_{\pi}(t) \beta_{q} \frac{2 m_{\pi}^{2} f_{\pi q q}^{2}}{2 m_{q}^{2}-m_{\pi}^{2} / 2-t} F_{\mathbb{P} q q}(t) \\
& \times 3 F_{1}(t) \beta_{q^{\prime}} \bar{u}\left(p^{\prime}\right)(\not k+q) u(p) \mathcal{R}^{\mathbb{P}}(s, t),
\end{aligned}
$$

where $F_{\pi}(t) \beta_{q^{\prime}} \gamma^{\mu}$ and $F_{1}(t) \beta_{q^{\prime}} \gamma_{\mu}$ with $\beta_{u}=2.07 \mathrm{GeV}^{-1}$ and $\beta_{d}=\beta_{u}$ are the Pomeron couplings to a quark in the pion and in the nucleon as discussed in Eq. (35). The form factor [32]

$$
F_{\mathbb{P} q q}(t)=\frac{2 \mu_{0}^{2}}{2 \mu_{0}^{2}+2 m_{q}^{2}-m_{\pi}^{2} / 2-t}
$$

is included to ensure the convergence of the off-shell quark loop with the cutoff mass $\mu_{0}^{2}=1.1 \mathrm{GeV}^{2}$ fixed to experimental data [34].

Another newly included quantity is the coupling constant $f_{\pi q q}$, which is expected to obey the Goldberg-Treiman relation at the quark level as

$$
\frac{f_{\pi q q}}{2 m_{q}}=\frac{1}{2 f_{\pi}} \frac{3}{5} g_{A} .
$$

Given the nucleon axial charge $g_{A}=1.25$, pion decay constant $f_{\pi}=93.1 \mathrm{MeV}$, and by using the quark mass $m_{q}=$ $330 \mathrm{MeV}$ we determine $f_{\pi q q}=2.65$.

It is worth noting in Eq. (42) that $\left(t / 2+k^{2} / 2-\right.$ $\left.q^{2} / 4-m_{q}^{2}\right)^{-1}$ becomes singular near $-t \approx 0$ as $k^{2}=q^{2}=$ $m_{\pi}^{2}$ for the pion elastic scattering when $m_{\pi}=2 m_{q}$ is assumed. For a better convergence of the quark loop in addition to the form factor $F_{\mathbb{P} q q}(t)$, therefore, we utilize the pion form factor $F_{\pi}(t)$. Moreover, in order to adjust the range of the $F_{\pi}(t)$ it is convenient to use the cutoff mass in Eq. (37), having an energy dependence as

$$
\Lambda(k)=\frac{k}{\mu}\left(W-W_{t h}\right),
$$

where $k$ is the incident pion momentum in the c.m. system, $\mu$ is the parameter of mass unit, and $W_{t h}$ is the total energy at threshold.

Figure 6 shows the divergence of the Pomeron exchange depending on the quark mass, for instance, $m_{q}=140 \mathrm{MeV}$ used without the form factor $F_{\pi}(t)$. Dotted, dash-dotted, and

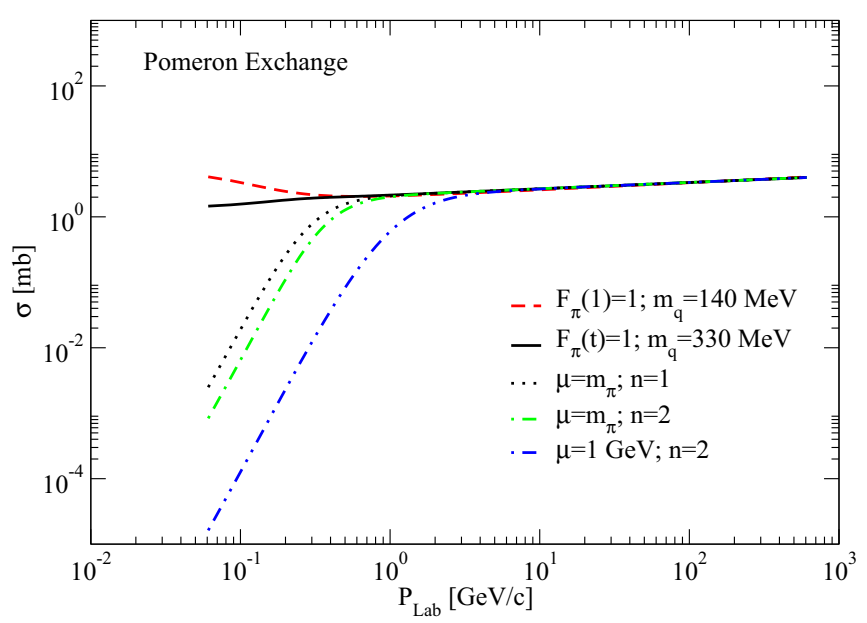

FIG. 6. Dependence of Pomeron exchange on $F_{\pi}(t)$. Given the physical pion mass $m_{\pi}$ and Pomeron trajectory in Eq. (47), the red dashed curve shows the divergence for $m_{q}=140 \mathrm{MeV}$ in the absence of $F_{\pi}(t) . f_{\pi q q}=1.32$ is used for a coincident with others for comparison. The rest of curves are resulting from the change of mass parameter $\mu$ and power $n$ with $m_{q}=330 \mathrm{MeV}$ fixed.

dash-dot-dotted curves are the cases of the Pomeron converging in the lower energy region due to the role of $F_{\pi}(t)$ with the parameter $\mu$ and power $n$ as designated in the figure.

Figure 7 presents differential cross sections for $\pi^{+} p$ and $\pi^{-} p$ elastic scatterings. In each reaction those cross sections at high momenta $P_{\mathrm{Lab}}=100$ and $200 \mathrm{GeV} / c$ in the upper two panels are used to determine the Pomeron trajectory, while all

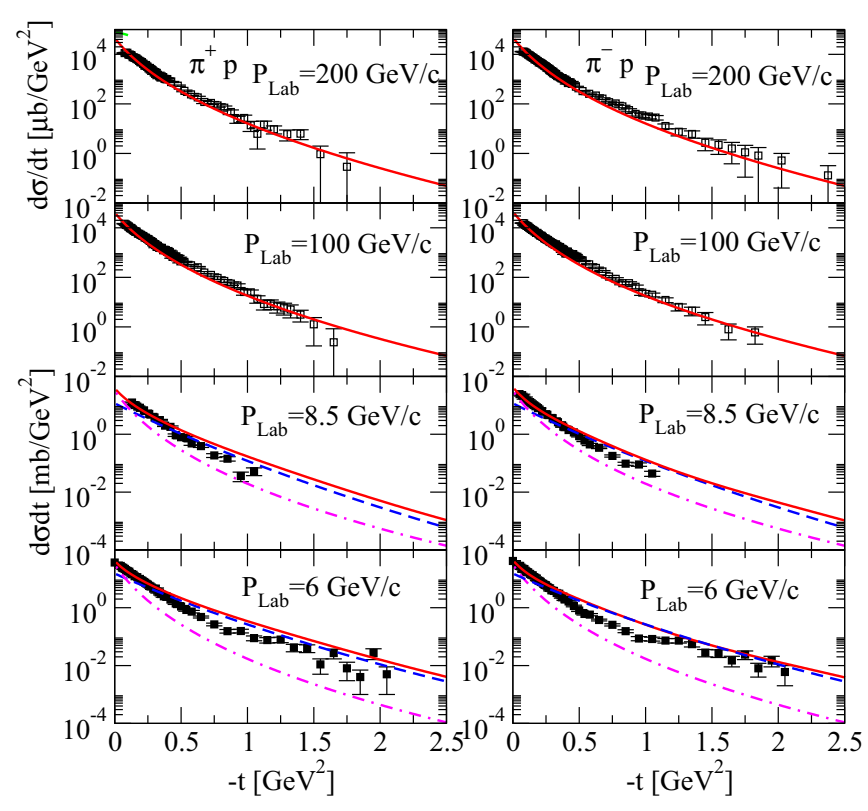

FIG. 7. Differential cross sections $d \sigma / d t$ for $\pi^{+} p$ (left) and $\pi^{-} p$ (right) elastic scattering at $P_{\mathrm{Lab}}=200,100,8.5$, and $6 \mathrm{GeV} / c$, respectively. Dashed and dash-dotted curves in the lower two panels are the contributions of $f_{2}$ and Pomeron. Data at 100 and $200 \mathrm{GeV} / c$ pion momenta are taken from Ref. [35] and data at $6.08 .5 \mathrm{GeV} / c$ are from Refs. [36,37]. 


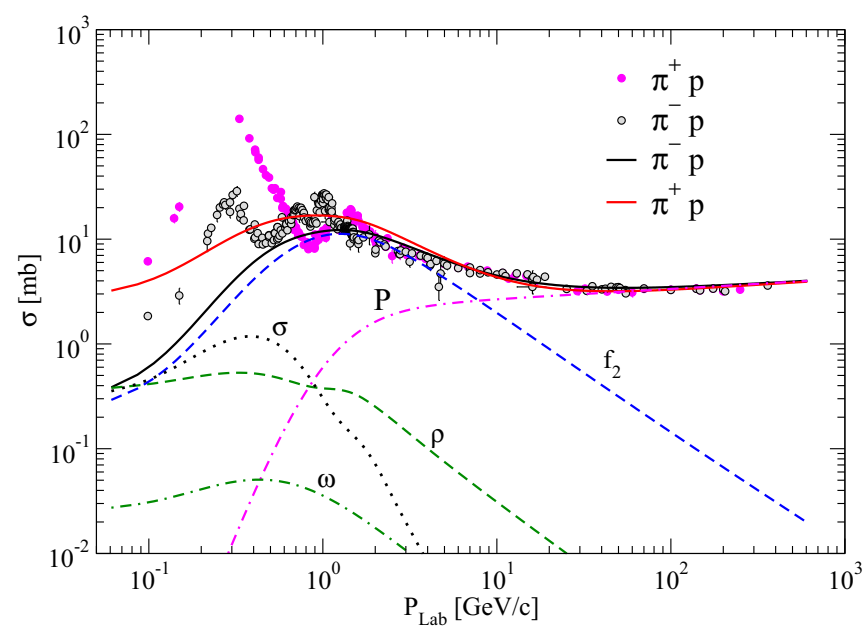

FIG. 8. Total cross section $\sigma$ for elastic reactions $\pi^{+} p$ (red) and $\pi^{-} p$ (black). Notations for $f_{2}$ and Pomeron are the same as in Fig. 7 for both processes. The difference between $\pi^{+} p$ and $\pi^{-} p$ cross sections is due to the roles of $\rho+\omega$ exchanges. Dominance of $f_{2}$ + Pomeron exchanges is apparent. World data are taken from the PDG [38].

the physical constants we use for $f_{\pi q q}, \beta_{u}, \beta_{d}$, and $\mu_{0}^{2}$ are fixed as before. Nevertheless, there is no criterion for what value we have to choose for the parameter $\mu$ at present, because the existing data are insensitive to a change of $\mu$. In this work we choose $\mu=1 \mathrm{GeV}$ and $n=2$ for illustration purposes. Then, by leaving the slope and intercept of the Pomeron trajectory $\alpha_{\mathbb{P}}(t)$ free parameters to fit to high-energy data, we obtain a good agreement with the energy and $t$ dependence of the cross sections at the choice of

$$
\alpha_{\mathbb{P}}(t)=0.12 t+1.06
$$

for Eq. (39). We note that the slope in the $\pi N$ scattering is consistent with Ref. [8], but by the factor of $1 / 2$ slower than that of the Pomeron $\alpha_{\mathbb{P}}(t)=0.25 t+1.08$ fitted to the total cross section of the $\pi N$ reaction [27]. Note that the slope of the total cross section at high energies given as the energy to the power $\sim s^{0.0808}$ [thus, $\alpha(0)=1.08$ by $\sigma \simeq s^{\alpha(0)-1}$ ] is by far different from that of the elastic cross section of the present case, as can be seen in Fig. 8.

In Fig. 8 we present total elastic cross sections for $\pi^{+} p$ and $\pi^{-} p$, where the contributions of the meson exchange as well as that of the Pomeron are shown. A few remarks are in order on the features of meson exchanges. The vector mesons $\rho$ and $\omega$ are responsible for the difference between $\pi^{+} p$ and $\pi^{-} p$ cross sections, as shown from threshold up to $P_{\mathrm{Lab}} \approx 2 \mathrm{GeV} / c$. At high momenta, the exchanges of $f_{2}$ and the Pomeron in the isoscalar channel are dominant over $\rho$ and $\omega$ so that the two cross sections coincide with each other, which should be distinguished from the difference between the total cross sections at high energy as in Refs. [6,26,32]. Thus, the reaction mechanisms of $\pi^{ \pm} p$ elastic reactions are characterized by the dominance of the natural parity exchange in the isoscalar channel.

Polarization of the target proton is the observable that could verify the accuracy of model predictions for the experimental

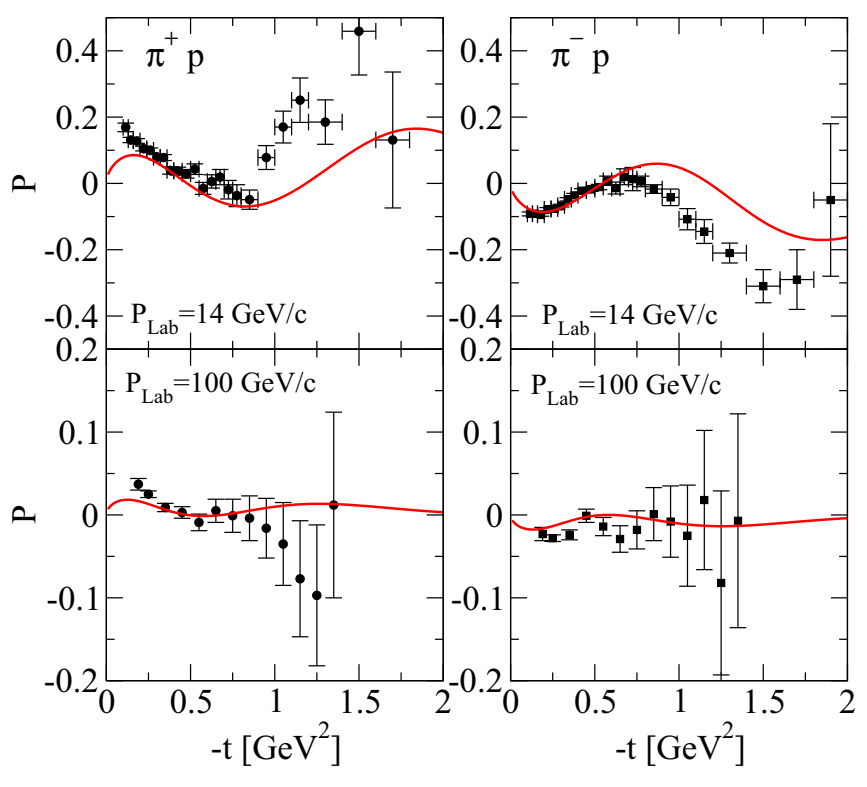

FIG. 9. Polarization asymmetry $P(t)$ for $\pi^{+} p$ (left) and $\pi^{-} p$ (right) elastic scattering at $P_{\mathrm{Lab}}=14$ and $100 \mathrm{GeV} / c$. Predictions from the model are in good agreement with data. Data are taken from Refs. [39,40].

measurement. To show the validity of the present model we present the polarization for $\pi^{ \pm} p$ reactions in Fig. 9 at intermediate and high momenta $P_{\text {Lap }}=14$ and $100 \mathrm{GeV} / c$. In these results the mirror symmetry between $\pi^{+} p$ and $\pi^{-} p$, which is a feature of polarizations of opposite charge, is well reproduced in any momentum range. In particular, polarizations are sensitive to the contribution of $f_{2}$ exchange with the coupling constant $g_{f_{2} N N}^{(2)}=0$, for better agreement with data.

\section{BARYON RESONANCES BELOW $W \leqslant 2 \mathrm{GeV}$}

In this section we present the nucleon resonances in the energy dependence of the cross section based on the $t$-channel exchanges as discussed in previous sections. More data from the angular distributions and spin polarizations could make the resonance parameters more precise. The most updated analysis for the nucleon resonances can be found in the SAID program of Ref. [41]. However, such a fine-tuning is beyond the scope of the present work and our aim here is to demonstrate how the Regge poles are well suited for the nucleon resonance of the Breit-Wigner form in the reaction amplitude,

$$
\mathcal{M}=\left(\mathcal{M}_{\text {Regge }}+\mathcal{M}_{\mathbb{P}}\right)+\mathcal{M}_{R}
$$

By the conventional definition of the nonrelativistic scattering amplitude as in the Appendix, we write the scattering amplitude as

$$
\mathcal{M}_{R}=\frac{8 \pi W}{\sqrt{4 M M^{\prime}}} \sqrt{\frac{k}{q}}[F(s, \theta)+i \sigma \cdot \hat{n} G(s, \theta)],
$$




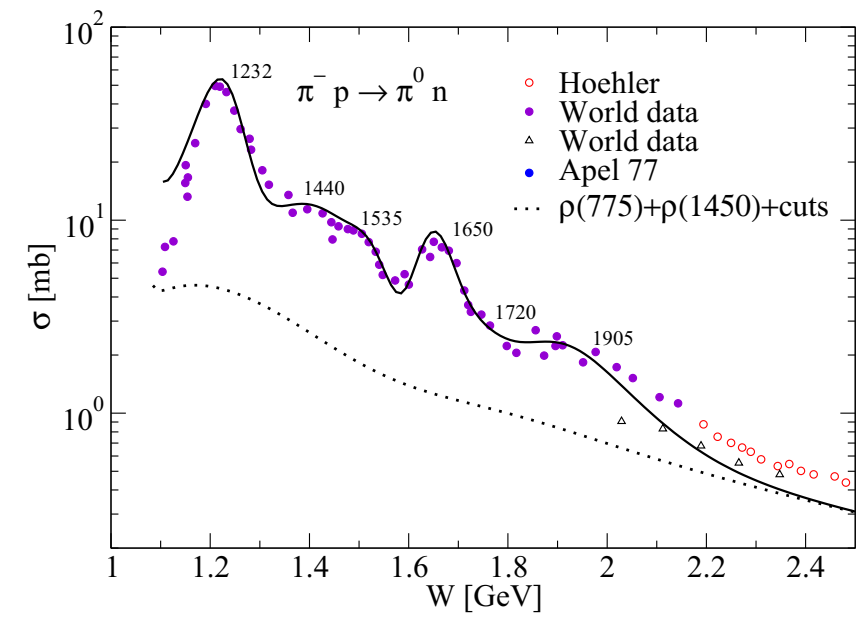

FIG. 10. Nucleon resonances in the $\pi^{-} p \rightarrow \pi^{0} n$ reaction.

with $\hat{n}=\hat{k} \times \hat{q} / \sin \theta$, and consider the spin nonflip and flip amplitudes to be of the form [42]

$$
\begin{aligned}
& F(s, \theta)=\frac{1}{k} \sum_{R} \frac{c_{R}\left(J_{R}+1 / 2\right)}{\epsilon_{R}-i} e^{-d \epsilon_{R}^{2}} P_{l}(\cos \theta), \\
& G(s, \theta)=\frac{1}{k} \sum_{R} \frac{c_{R}(-1)^{J_{R}-l+1 / 2}}{\epsilon_{R}-i} e^{-d \epsilon_{R}^{2}} \frac{d P_{l}(\cos \theta)}{d \cos \theta},
\end{aligned}
$$

with the $d$ in the Gaussian type of the damping factor to adjust the width of the resonance. Here, $c_{R}=I_{R} X_{R}$ is a sort of coupling strength of the resonance $R$ originating from the product of the Clebsch-Gordon coefficient for isospin and elasticity. $\epsilon_{R}=\left(M_{R}^{2}-s\right) / M_{R} \Gamma_{R}$ is the $s$-channel pole with the mass and full width of the resonance $R . k$ and $\theta$ are the momentum and scattering angle in the c.m. system. $J_{R}$ is the spin of the resonance.

Figure 10 shows the total cross section for $\pi^{-} p \rightarrow \pi^{-} n$, in which case the $t$-channel meson exchanges in Eq. (16) constitute a background contribution upon which nucleon resonances are mounting. Nucleon resonances $\Delta(1232)$, $N^{*}(1440), N^{*}(1535), N^{*}(1650), N^{*}(1720)$, and $\Delta(1905)$ are introduced with their parameters fitted to the total cross section data as in Table II. Note that, unlike in Ref. [43], the contributions of the $t$-channel exchanges in the present calculation are not passing through the average of the cross section on the energy interval below $W \approx 2 \mathrm{GeV}$, and we expect that the problem of double counting should be insignificant.

The resonance structures in $\pi^{-} p \rightarrow \pi^{-} p$ and $\pi^{+} p \rightarrow$ $\pi^{+} p$ reactions are presented in Figs. 11 and 12. It is worth remarking that the scalar meson coupling constant $f_{\sigma \pi \pi}=$ -0.5 is used with its sign reversed, because it is advantageous to alleviate the problem of double counting by reducing the contribution of the $\sigma$ meson, which may overlap with a resonance. On the other hand, we have to neglect the threshold divergence of the $\Delta$ pole in Figs. 10 and 11 which are not covered up by the Gaussian damping factor in the multipoles. In practice, this is a drawback of the present model calculation of the resonances, formulated as in Eqs. (50) and (51). Moreover, in the case of the $\pi^{+} p \rightarrow \pi^{+} p$ reaction where we
TABLE II. $\Delta$ and $N^{*}$ resonances in $\pi N$ scatterings. Mass and width in units of $\mathrm{MeV}$ are taken at the Breit-Wigner fit in the PDG. Process I stands for $\pi^{-} p \rightarrow \pi^{0} n$, II for $\pi^{-} p \rightarrow \pi^{-} p$, and III for $\pi^{+} p \rightarrow \pi^{+} p$, respectively.

\begin{tabular}{cccccc}
\hline \hline Process & Resonance & $M_{R}$ & $\Gamma_{R}$ & $c_{R}$ & $d$ \\
\hline I & $\Delta^{0}(1232) P_{33}$ & 1232 & 125 & 0.5 & 0.3 \\
& $N^{*}(1440) P_{11}$ & 1440 & 400 & 0.6 & 0.7 \\
& $N^{*}(1535) S_{11}$ & 1510 & 150 & 0.4 & 0.5 \\
& $N^{*}(1650) S_{11}$ & 1650 & 125 & 0.7 & 0.4 \\
& $N^{*}(1720) P_{13}$ & 1720 & 250 & 0.3 & 0.4 \\
& $\Delta^{0}(1905) F_{35}$ & 1900 & 300 & 0.2 & 0.1 \\
II & $\Delta^{0}(1232) P_{33}$ & 1232 & 125 & 0.35 & 0.4 \\
& $N^{*}(1440) P_{11}$ & 1420 & 400 & -0.5 & 2.2 \\
& $N^{*}(1535) S_{11}$ & 1510 & 150 & 0.6 & 0.5 \\
& $N^{*}(1650) S_{11}$ & 1650 & 125 & 0.75 & 0.4 \\
& $N^{*}(1720) P_{13}$ & 1720 & 250 & 0.3 & 0.4 \\
& $\Delta^{0}(1905) F_{35}$ & 1900 & 300 & 0.2 & 1 \\
III & $\Delta^{++}(1232) P_{33}$ & 1235 & 120 & 2 & $0.03^{\text {a }}$ \\
& $\Delta^{++}(1905) F_{35}$ & 1900 & 400 & 0.5 & 0.9 \\
\hline \hline
\end{tabular}

a In III, in addition to the Gaussian damping factor with the parameter $d$, the cutoff function in Eq. (37) with $n=1$ and $\mu=m_{\pi}$ is applied to the $\Delta^{++}$multipole.

have to reproduce the $\Delta^{++}(1232)$ pole with such a wide width that amounts to $500-600 \mathrm{MeV}$ as can be seen in Fig. 12, the threshold divergence is even worse. In order to suppress the strong divergence near threshold, we apply the cutoff function in Eq. (37) for the $\Delta^{++}$(1232) pole with $n=1$ and $\mu=m_{\pi}$ in Eq. (46), in addition to the Gaussian damping factor.

\section{SUMMARY AND CONCLUSIONS}

In this work we have investigated $\pi^{-} p \rightarrow \pi^{0} n$ charge exchange and $\pi^{ \pm} p \rightarrow \pi^{ \pm} p$ elastic reactions up to incident pion momentum $P_{\mathrm{Lab}} \approx 250 \mathrm{GeV} / c$ to provide a theoretical framework that could validate a consistency of the coupling strengths and forms of interaction Lagrangians between

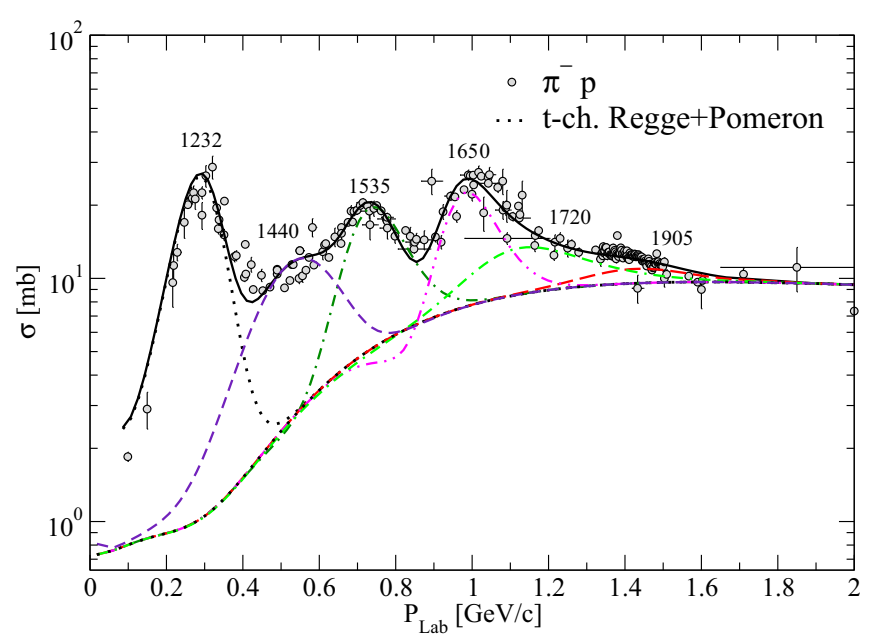

FIG. 11. Nucleon resonances in the $\pi^{-} p \rightarrow \pi^{-} p$ reaction. The respective contributions of nucleon resonances are presented. 


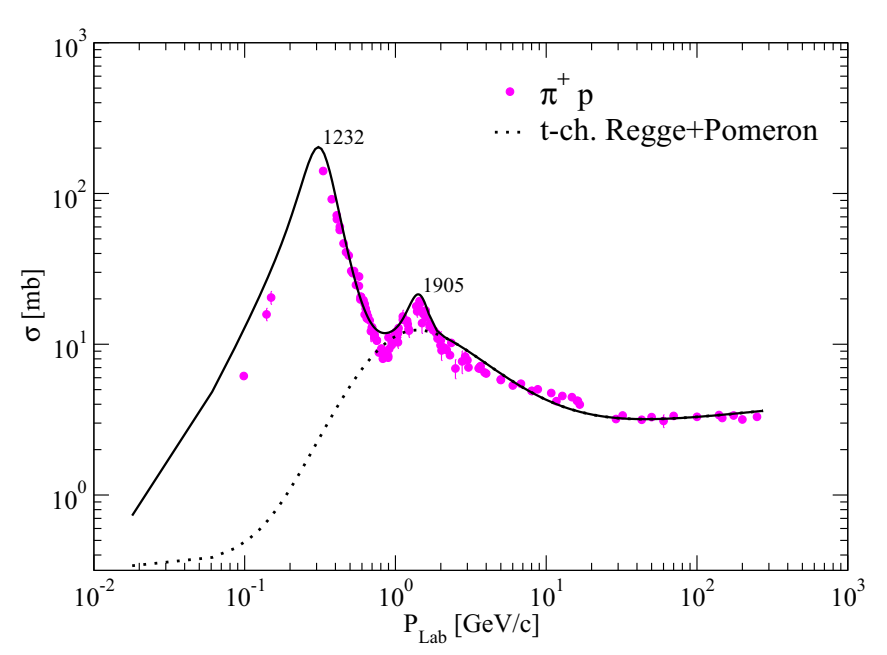

FIG. 12. Nucleon resonances in the $\pi^{+} p \rightarrow \pi^{+} p$ reaction. Resonances $\Delta(1232)+\Delta(1905)$ reproduce the peaks.

hadrons at low and high energies. For a description of the reaction in the Regge realm we utilize the relativistic Born amplitude for the Reggeization of the $t$-channel meson exchange. Through the reproduction of reaction cross sections, the reaction mechanisms by the $t$-channel meson and Pomeron exchanges are analyzed, with the coupling constants for hadron interactions shared with other hadron reactions, e.g., photoproductions of vector mesons.

A unique role of vector meson $\rho$ (775) in the charge exchange reaction is investigated. Given the single $\rho$ exchange with the deep dip at the NWSZ point $-t=0.51 \mathrm{GeV}^{2}$, the dip-filling mechanism for the differential cross section needs $\rho-f_{2}$ and $\rho$-Pomeron cuts. In order to reproduce the spin polarization a second $\rho(1450)$ Regge pole is called for, with the trajectory predicted from the relativistic quark model, though the coupling constants of the $\rho(1450)$ are treated as free parameters. These theoretical entities yield the Regge description of the charge exchange process to a good degree.

The exchange of a soft Pomeron is newly constructed from the quark-Pomeron coupling picture and applied successfully for $\pi^{ \pm} p$ elastic scatterings with a trajectory quite different from that from the total cross sections for the $\pi N$ reaction. The difference between the $\pi^{+} p$ and $\pi^{-} p$ elastic cross sections is insignificant because of the minor roles of $\rho+\omega$ exchanges, while $f_{2}(1275)$ and Pomeron exchanges are dominant in the overall range of pion momentum. Polarizations are well reproduced with the mirror symmetry reflected between the two reactions of opposite charges.

Nucleon resonances below $W \leqslant 2 \mathrm{GeV}$ are reproduced in three channels, $\pi^{-} p \rightarrow \pi^{0} n, \pi^{+} p \rightarrow \pi^{+} p$, and $\pi^{-} p \rightarrow \pi^{-} p$, and they are consistent with existing data within the masses, widths, and branching fractions reported in the PDG. These findings illustrate how the $t$ channel Regge poles in the present framework do well for the analysis of nucleon resonances in the low-energy region as well as the description of the reactions at high energies.

\section{ACKNOWLEDGMENTS}

This work was supported by the National Research Foundation of Korea Grant No. NRF2017R1A2B4010117 and partially funded by Grant No. NRF-2016K1A3A7A09005580.

\section{APPENDIX: PARTIAL WAVE EXPANSION FOR NUCLEON RESONANCE} by

The scattering amplitude in the $\pi N$ c.m. system is defined

$$
\frac{\sqrt{4 M M^{\prime}}}{8 \pi W} \mathcal{M}=\chi^{\dagger}[F(s, \theta)+i \sigma \cdot \hat{n} G(s, \theta)] \chi
$$

with our convention for the normalization constant $N=$ $\sqrt{\frac{E+M}{2 M}}$ for the Dirac spinor. Here $\chi$ is the $2 \times 1$ Pauli spinor with spin and isospin indices understood.

The differential cross section is calculated by the equation

$$
\frac{d \sigma}{d \Omega}=\frac{q}{k}\left|\frac{\sqrt{4 M M^{\prime}}}{8 \pi W} \mathcal{M}\right|^{2}=|F|^{2}+\sin ^{2} \theta|G|^{2} .
$$

The spin nonflip and flip parts of the scattering amplitude are expanded with the orbital momentum $l$ and the total angula momentum $J$,

$$
\begin{aligned}
& F(s, \theta)=\sum_{l=0}\left[(l+1) f_{l+}(s)+l f_{l-}(s)\right] P_{l}(\cos \theta), \\
& G(s, \theta)=\sum_{l=1}\left[f_{l+}(s)-f_{l-}(s)\right] \frac{d P_{l}(\cos \theta)}{d \cos \theta} .
\end{aligned}
$$

Each partial wave of $\alpha(=l \pm)$ is related to the phase shift by

$$
f_{\alpha}(s)=\frac{1}{2 i k}\left(e^{2 i \delta_{\alpha}}-1\right) .
$$

The energy dependences of the partial wave for the spin nonflip and flip amplitudes in Eqs. (A3) and (A4) are parametrized as in Eqs. (50) and (51).
[1] D. Siemens, V. Bernard, E. Epelbaum, A. M. Gasparyan, H. Krebs, and Ulf-G. Meißner, Phys. Rev. C 96, 055205 (2017).

[2] R. A. Arndt, W. J. Briscoe, I. I. Strakovsky, R. L. Workman, and M. M. Pavan, Phys. Rev. C 69, 035213 (2004).

[3] R. A. Arndt, W. J. Briscoe, I. I. Strakovsky, and R. L. Workman, Phys. Rev. C 74, 045205 (2006).
[4] R. Koch and E. Pietarinen, Nucl, Phys. A 336, 331 (1980).

[5] M. A. Pichowsky and T.-S. H. Lee, Phys. Rev. D 56, 1644 (1997).

[6] V. Mathieu, I. V. Danilkin, C. Fernández-Ramírez, M. R. Pennington, D. Schott, A. P. Szczepaniak, and G. Fox, Phys. Rev. D 92, 074004 (2015). 
[7] J. Nys, A. N. Hiller Blin, V. Mathieu, C. Fernández-Ramírez, A. Jackura, A. Pilloni, J. Ryckebusch, A. P. Szczepaniak, and G. Fox, Phys. Rev. D 98, 034020 (2018).

[8] F. Huang, A. Sibirtsev, S. Krewald, C. Hanhart, J. Haidenbauer, and U.-G. Meißner, Eur. Phys. J. A 40, 77 (2009).

[9] C. T. Hung, S. N. Yang, and T.-S. H. Lee, J. Phys. G 20, 1531 (1994).

[10] A. Donnachie and P. V. Landshoff, Nucl. Phys. B 244, 322 (1984).

[11] J.-M. Laget and R. Mendez-Galain, Nucl. Phys. A 581, 397 (1995).

[12] B.-G. Yu, H. Kim, and K.-J. Kong, Phys. Rev. D 95, 014020 (2017).

[13] B.-G. Yu and K.-J. Kong, arXiv:1710.04511.

[14] A. Donnachie and Yu. S. Kalashnikova, Phys. Rev. C 93, 025203 (2016).

[15] A. I. Titov and T.-S. H. Lee, Phys. Rev. C 66, 015204 (2002).

[16] Y. Oh, A. I. Titov, and T.-S. H. Lee, Phys. Rev. C 63, 025201 (2001).

[17] B.-G. Yu and K.-J. Kong, Phys. Lett. B 765, 221 (2017).

[18] B.-G. Yu and K.-J. Kong, arXiv:1612.02071.

[19] A. V. Barnes, D. J. Mellema, A. V. Tollestrup, R. I. Walker, O. I. Dahl, R. A. Johnson, R. W. Kenney, and M. Pripstein, Phys. Rev. Lett. 37, 76 (1976).

[20] Y. Oh and T.-S. H. Lee, Phys. Rev. C 69, 025201 (2004).

[21] J. M. Laget, Phys. Lett. B 695, 199 (2011).

[22] S. J. Brodsky, G. F. de Téramond, and H. Günter Dosch, arXiv:1302.5399.

[23] D. Ebert, R. N. Faustov, and V. O. Galkin, Phys. Rev. D 79, 114029 (2009).

[24] D. Hill, P. Koehler, T. Novey, P. Rynes, B. Sandler, H. Spinka, A. Yokosawa, D. Eartly, K. Pretzl, G. Burleson, G. Hicks, C. Wilson, and W. Risk, Phys. Rev. Lett. 30, 239 (1973).

[25] W. D. Apel et al., Nucl. Phys. B 154, 189 (1979).

[26] R. J. N. Phillips and W. Rarita, Phys. Rev. 139, B1336 (1965).
[27] A. Donnachie and P. V. Landshoff, Phys. Lett. B 296, 227 (1992).

[28] A. V. Friesen, Yu. L. Kalinovsky, and V. D. Toneev, Phys. Part. Nuclei Lett. 9, 1 (2012).

[29] N. Wu, arXiv:hep-ex/0104050.

[30] C. Schütz, J. W. Durso, K. Holinde, and J. Speth, Phys. Rev. C 49, 2671 (1994).

[31] B.-G. Yu, T. K. Choi, and W. Kim, Phys. Lett. B 701, 332 (2011).

[32] S. Donnachie, G. Dosch, P. Landshoff, and O. Nachtmann, Pomeron Physics and QCD (Cambridge University Press, Cambridge, 2002).

[33] A. I. Titov, Y. Oh, S. N. Yang, and T. Morii, Phys. Rev. C 58, 2429 (1998).

[34] A. Donnachie and P. V. Landshoff, Phys. Lett. B 185, 403 (1987).

[35] C. W. Akerlof, R. Kotthaus, R. L. Loveless, D. I. Meyer, I. Ambats, W. T. Meyer, C. E. W. Ward, D. P. Eartly, R. A. Lundy, S. M. Pruss, D. D. Yovanovitch, and D. R. Rust, Phys. Rev. D 14, 2864 (1976).

[36] I. Ambats, D. S. Ayres, R. Diebold, A. F. Greene, S. L. Kramer, A. Lesnik, D. R. Rust, C. E. W. Ward, A. B. Wicklund, and D. D. Yovanovitch, Phys. Rev. D 9, 1179 (1974).

[37] D. Harting et al., Nuovo Cimento 38, 60 (1965).

[38] C. Amsler et al. (Particle Data Group), Phys. Lett. B 667, 1 (2008).

[39] M. Borghini et al., Phys. Lett. B 36, 493 (1971).

[40] I. P. Auer, D. Hill, B. Sandler, A. Yokosawa, W. Brückner, O. Chamberlain, H. Steiner, G. Shapiro, A. Jonckheere, P. F. M. Koehler, R. V. Kline, M. E. Law, F. M. Pipkin, W. Johnson, J. Snyder, and M. E. Zeller, Phys. Rev. Lett. 39, 313 (1977).

[41] R. L. Workman, R. A. Arndt, W. J. Briscoe, M. W. Paris, and I. I. Strakovsky, Phys. Rev. C 86, 035202 (2012).

[42] A. J. Lennox et al., Phys. Rev. D 11, 1777 (1975).

[43] R. Dolen, D. Horn, and C. Schmid, Phys. Rev. 166, 1768 (1968). 